\title{
Off-target genome editing: A new discipline of gene science and a new class of medicine
}

\author{
Diane Catherine Wang • Xiangdong Wang
}

Received: 8 April 2019 /Accepted: 18 April 2019 / Published online: 27 April 2019

(C) Springer Nature B.V. 2019

\begin{abstract}
With an increasing growth of genome editing, off-target effects such as non-specific genetic modifications resulting from the designed process of genome editing become a new discipline of gene science and new class medicine. The degree of short-term and long-term side effects and toxicity or dynamics of the primary and secondary off-target genome editing varies with the application of different methodologies of gene editing and measuring, readouts of genetic modifications, or comparison reference. Measurements of dynamic off-target effects caused directly or indirectly by genome editing are critical in clinical application of gene editing. The quality of genome editing methods is one of the decisive factors in the occurrence of off-target effects. Mechanisms by which off-target effects of genome editing occurs are more complex and comprehensive than we expected. The heterogeneity of off-target effects of gene-edited cells at single-cell levels should be defined during the development and formation of cell clusters. In addition to off-target effects on gene-edited cells per se, alterations of gene sequence, structure, dimension, and function of related regulators caused by off-target effects may also influence intercellular communications and interactions between gene-edited cells, between gene-edited cells and non-edited cells, or
\end{abstract}

D. C. Wang $\cdot X$. Wang $(\bowtie)$

Zhongshan Hospital Institute for Clinical Science, Shanghai Institute of Clinical Bioinformatics, Shanghai Engineering Research for AI Technology for Cardiopulmonary Diseases, Shanghai Medical College, Fudan University, Shanghai, China e-mail: xdwang@fuccb.com between non-edited cells. Thus, controlling, measuring, defining, categorizing, and predicting off-target genome editing need to be standardized and prioritized before clinical application of gene editing.

Keywords Gene editing · Off-target - Therapy · Toxicity $\cdot$ Side effects

With rapid development and improvement of gene editing technologies, a large number of new therapeutic concepts have appeared for clinical considerations, e.g., gene therapy, genome editing, or nucleic acid therapeutics. A new class of medicines will be generated from a series of consequences after clinical application of genome editing (Porteus 2019). With increasing precision editing, transplantation of genome-engineered cells and embryonic cells may be an alternative for the prevention and treatment of genetic and nongenetic diseases. It seems that genome editing can prevent and cure any disease as long as the disease-specific target gene is identified and validated. Thus, the three most critical and limiting factors to push genome editing into clinical applications are the disease specificity of target gene, the therapeutic efficacy to improve disease severity, and the safety and toxicity of gene editing procedures and materials. However, there are a large number of challenges to be overcome before genome editing can be applied to patients. For example, we need clear and evidencebased criteria and standards to allow ethical permission for genome editing, show target gene disease-specificity, and ensure safety of the process (Fang and Wang 
2016; Sakuma and Yamamoto 2017; Wang et al. 2018). Of those challenges, off-target genome editing should be paid more attention to, since the off-target effects are not dynamically monitored, qualitied, quantified, or anticipated. There is little known about the short-term and long-term side-effects and toxicity of off-target genome editing in humans. Off-target genome editing will be a new discipline of gene science and a new class of medicine.

The concept of off-target genome editing should be redefined by the rapid growth of knowledge and experience on analysis of off-target genes and their effects. Off-target is defined as non-specific genetic modifications as a result of a designed genome editing process, including point mutations, deletions, insertions, inversions, and translocations of unexpected genes. Those are countable effects of editing techniques per se, while the long-term biological effects are not estimated. It is questioned whether the non-specific genetic modification caused by designed and selected target genes should also count as off-target genome editing, as those genes are expected, intended, designed, and targeted. Such non-specific genetic modification may be caused by the complexity of disease, lack of systemic validation of identified targets, and unique focus on biological effects rather than pathophysiological specificity. For example, over-expression of CCR5 gene and protein was found in people with HIV infection, and the allogeneic hematopoietic stem-cell transplantation procedures with a homozygous mutation in the HIV coreceptor CCR5 (CCR5 $\Delta 32 / \Delta 32)$ or gene editing of CCR5 in autologous CD4 T cells could remiss HIV-1 expression in patients infected with HIV (Gupta et al. 2019; Tebas et al. 2014). Over-expression of CCR5 gene and protein can occur in multiple pathological conditions and the existence of CCR5 is critical and necessary to maintain the normality of native immune function. There is no answer to how many off-target genes will be caused by the embryonic CCR5 gene editing to prevent the occurrence of HIV infection or what consequences will result from the secondary off-target effects.

Dynamic effects of the primary and secondary offtarget genome editing should be emphasized and paid more attention to. For example, transplantation of geneedited cells has been suggested as an alternative therapy for diseases. Gene-edited immune cells can be used for leukemias, lymphomas, solid tumors, and HIV infection. Sharpe (2018) overviewed and demonstrated a large number of predicted and unpredicted toxicities, including off-tumor activity, cytokine release syndromes, neurotoxicity, and fatal consequences during or after gene-edited $\mathrm{T}$ cell therapies. In addition, it remains unclear whether the gene editing causes offtarget effects on gene-edited cells per se, which may extend or secondarily occur within the gene-edited cell, or whether gene-edited cells can result in the "infected" off-target effects in other non-gene-edited host cells. A similar challenge should be considered in gene-edited stem cells of which therapeutic effects are confirmed for tissue repair, organ dysfunction, and regeneration. Gene-edited stem cells also experience doublestranded chromosomal break and DNA repair through non-homologous end joining and homologous recombination, to achieve target-specific gene modifications. Stem cells have strong capacities for differentiation, proliferation, and formation of new clusters of geneedited cells, during which new biological phenomes will be generated, while off-target double-stranded chromosomal breaks and non-specific genetic modifications are simultaneously or secondarily created, regardless of whether the designed sequence binds to the target or not. Veres et al. (2014) studied the degree of genomewide off-target mutagenesis in ten cell lines of human pluripotent stem cells targeted with either clustered, regularly interspaced, short palindromic repeats (CRISPR)-Cas9 or transcription activator-like effector nucleases (TALENs) using whole-genome sequencing at high coverage $(60 \times$ target coverage). They proposed that nuclease-mediated off-target effects occurred in gene-edited cells at limited amounts might be not severe enough to obstruct clinical application of stem cells.

The degree of off-target effects reported varies due to the application of different analytic methodologies, focused readouts of genetic modifications, comparison reference, as well as editing methods. Veres et al. (2014) found minimal amount of off-target effects based on detection of off-target insertions and deletions by comparing with the parental cells, while Kosicki et al. (2018) reported significant on-target mutagenesis according to large deletions and more complex genomic rearrangements at the targeted sites in mouse embryonic stem cells, mouse hematopoietic progenitors and a human differentiated cell line. Using conventional Sanger sequencing technology, Kosicki et al. (2018) found $74 \%$ simple deletions overlapping both the cut site and the exon, $17 \%$ recovered alleles containing additional lesions, $57 \%$ having only exonic lesion, and $26 \%$ insertions in recovered alleles. The extensive and 
unexpected results off-target genomic editing have inevitable and common outcomes, which occur both at the target locus and beyond. There are no clear answers on what the severe long-term transcriptional consequences of the off-target genome editing are, whether those offtarget effects caused mutations that can induce new neoplasia, or whether off-target-associated invisible and detectable genetic changes will result in inherent toxicity or carcinogenesis for coming generations.

Measurements of off-target effects are the critical factor in clinical application of gene therapy. The emphasis will be to dynamically monitor off-target effects, precisely detect the sequence changes caused directly or indirectly by genome editing, and efficiently respond to concerns from clinicians and patients. One of the most difficult challenges is that we do not know what nonspecific genetic modifications may occur during/after genome editing, characteristics of off-target sequences to control the ratio between on-target and off-target effects, or the properties of the interaction between sequences and probes to design the optimal and most sensitive detectors. The customizable hybrid-capture and error-corrected ultra-deep circulating free DNASeq technology was suggested as a simple and sensitive method to detect duplex DNA-molecules for enhanced error correction, with a small amount of circulating free DNA and high detection rate of single nucleotide variants (Mansukhani et al. 2018). However, the potential for circulating free DNA to be used for measurements of off-target effects may still need further clarification and validation. The urgent challenge is to develop and optimize the consistency, reliability, and repeatability of qualitative and quantitative analysis to dynamically monitor those "unexpected" off-target effects. It is also necessary for society to identify and list details of offtarget effects, categorize severity of off-target effects, and explain the potential of biological influences, e.g., number, size, location of off-target genes, sequence property, pairing stability, designed-target rate, genetic interactions, and estimated lethality.

The quality of genome editing methods is a decisive factor in the occurrence of off-target effects. Jin et al. (2019) analyzed off-target effects between gene editing methods of adenine-base editor with cytosine-base editor (base editor 3 or high-fidelity base editor) in genetically modified rice and found that the adenine-base editor had less missed target genes. Zuo et al. (2019) developed a new genome-wide off-target analysis through two-cell embryo injection and found that cytosine-base editing induced off-target single nucleotide variants with over 20-fold higher frequencies than CRISPR-Cas9 or base editors. In this particular study, one blastomere of two-cell mouse embryos was edited with CRISPR-Cas9, a cytosine-base editor, and an adenine-base editor to compare the whole genome sequences of progeny cells of edited vs. non-edited blastomeres. Kim et al. (2019) found that CRISPR-Cas9, the cytosine-base editor, and the adenine-base editor had various levels of ability to define nucleotide mismatches and targeted different off-target sites. Those results deliver a clear message that the efficiency of detecting offtarget effects is highly dependent upon analytic methodologies, monitoring designs, measure protocols, comparison references, as well as species. The deepsequencing data demonstrated that near half of genome-editing projects confirmed about 1500 predicted off-target sites on mouse and rat genomes and that high-fidelity Cas9 versions reduced off-target mutation rates in vivo (Anderson et al. 2018).

The mechanisms behind how off-target effects of genome editing occur are more complex and comprehensive than expected. It therefore becomes more important to develop off-target effects-specific analyses to define the genome-wide specificities of each genome editing. This requires developing a method specific for an editing based on its recognition of off-target sites. Kim et al. (2019) declared that the genome-wide offtarget sites of CRISPR-Cas9, adenine-base editor and cytosine-base editor were identified with mismatched single guide RNAs for endogenous genomic loci, Digenome-seq for identifying CRISPR-induced double-strand breaks, and targeted amplicon sequencing for validation of cleavage sites. One of the outstanding outcomes from this particular study showed that adenine-base editor's off-target effects at defined sites could be avoided by the combination of Sniper adeninebase editor 7.10 with modified single guide RNAs. One of the editing method categories is the enzyme-based process during which targeted DNA double-stranded breaks are introduced into the genome followed by a homology-directed repair. The amount of off-target effects varies among nucleases, e.g., CRISPR-Cas9, TALEN, meganucleases, and zinc finger nucleases (ZFN). The key step error-prone nonhomologous end joining is a pathway to repair double-strand breaks in DNA through micro-homologous guide. Any imprecise or inappropriate repair will cause loss of nucleotides, insertion and deletion, or translocations and telomere 
fusion. Lin et al. (2018) developed CRISPR-guided DNA methyltransferases to methylate the $\mathrm{CpG}$ dinucleotides flanking its target sites at different genomic loci in human embryonic kidney cells. They found a number of the off-target effects in differentially methylated regions, promoter regions, 5' untranslated regions, $\mathrm{CpG}$ islands, and DNase I hypersensitivity sites, leading to significant off-target methylation.

Off-target effects of genome editing can be conceptualized as "off-target toxicity," a clinical measure, to evaluate or predict clinical risks and consequences of genome editing application. Trendowski et al. (2019) applied the concept of off-target toxicity to review the cytotoxicity of chemotherapy and demonstrate multiple off-target toxicities of cisplatin, as an effective measurement to identify individual susceptibility, genetic and nongenetic risk factors, and association between specific genotypes and drug-induced toxicities. In addition to sequencing, the off-target toxicity should be extended to structures and functions of DNA and associated regulators, RNA and RNA-associated factors, or genome dimensions and regulators, according to the new concept of clinical gene tests (Wu et al. 2019). The sensitivity, specificity, stability, analysis, and clinical significance of methodologies to monitor the off-target toxicity should be further defined to include standardizations, guidelines, and consortium of sample handling, experimental operation, quality control, data analysis, and clinical interpretation. We should clarify the contents of short-term or long-term effects, primary or secondary effects, structural or functional off-target toxicities, and develop predictive strategies for regular monitoring systems and regulations.

With the development of sequencing technology, a number of opportunities and challenges of single cell sequencing and analyzing need to be faced to discover new cell clusters and subtypes, intratissue heterogeneity, and new diagnostic biomarkers and therapeutic targets (Wang et al. 2018; Zhu et al. 2018). Of those, it is imperative to define the heterogeneity of off-target effects of gene-edited cells at single-cell levels, especially during the development and formation of cell clusters. In addition to off-target effects on gene-edited cells per se, alterations of gene sequence, structure, dimension, and function of related regulators caused by off-target effects may also influence intercellular communications and interactions between gene-edited cells, between gene-edited cells and non-edited cells, or between nonedited cells. Cell-to-cell communication is recently suggested as a new to understand the old mysteries of molecular mechanisms of the microenvironment complexity, heterogeneity, homeostasis, and function by profiling intercellular signal networks (Song et al. 2019).

In conclusion, off-target effects as non-specific genetic modifications resulted from the designed process of genome editing and will become a new discipline of gene science and a new class of medicine. The degree of short-term and long-term side-effects and toxicity or dynamics of the primary and secondary off-target genome editing varies with the application of different methodologies of gene editing and measuring, readouts of genetic modifications, or comparison reference. Measurements of dynamic off-target effects caused directly or indirectly by genome editing are critical in clinical application of gene editing. The quality of genome editing methods is one of the decisive factors in the occurrence of off-target effects. Mechanisms by which off-target effects of genome editing occurs are more complex and comprehensive than expected. The heterogeneity of off-target effects of gene-edited cells at single-cell levels should be defined during the development and formation of cell clusters. In addition to offtarget effects on gene-edited cells per se, alterations of gene sequence, structure, dimension, and function of related regulators caused by off-target effects may also influence intercellular communications and interactions between gene-edited cells, between gene-edited cells and non-edited cells, or between non-edited cells. Thus, controlling, measuring, defining, categorizing, and predicting the effects of off-target genome editing need to be standardized and prioritized before clinical application of gene editing.

\section{References}

Anderson KR, Haeussler M, Watanabe C, Janakiraman V, Lund J, Modrusan Z, et al. CRISPR off-target analysis in genetically engineered rats and mice. Nat Methods. 2018;15(7):512-4. https://doi.org/10.1038/s41592-018-0011-5.

Fang H, Wang W. Could CRISPR be the solution for gene editing's Gordian knot? Cell Biol Toxicol. 2016;32(6):4657. https://doi.org/10.1007/s10565-016-9359-4.

Gupta RK, Abdul-Jawad S, McCoy LE, Mok HP, Peppa D, Salgado M, et al. HIV-1 remission following CCR5 $\Delta 32$ / $\Delta 32$ haematopoietic stem-cell transplantation. Nature. 2019. https://doi.org/10.1038/s41586-019-1027-4.

Jin S, Zong Y, Gao Q, Zhu Z, Wang Y, Qin P, et al. Cytosine, but not adenine, base editors induce genome-wide off-target 
mutations in rice. Science. 2019. https://doi.org/10.1126 /science.aaw7166.

Kim D, Kim D, Lee G, Cho SI, Kim JS. RNA-guided adenine base editors. Genome-wide target specificity of CRISPR. Nature Biotechnol. 2019;37:430-5. https://doi.org/10.1038/s41587019-0050-1.

Kosicki M, Tomberg K, Bradley A. Repair of double-strand breaks induced by CRISPR-Cas9 leads to large deletions and complex rearrangements. Nat Biotechnol. 2018;36(8):765-71. https://doi.org/10.1038/nbt.4192.

Lin L, Liu Y, Xu F, Huang J, Daugaard TF, Petersen TS, et al. Genome-wide determination of on-target and off-target characteristics for RNA-guided DNA methylation by dCas9 methyltransferases. Gigascience. 2018;7(3):1-19. https://doi.org/10.1093/gigascience/giy011.

Mansukhani S, Barber LJ, Kleftogiannis D, Moorcraft SY, Davidson M, Woolston A, et al. Ultra-sensitive mutation detection and genome-wide DNA copy number reconstruction by error corrected circulating tumor DNA sequencing. Clin Chem. 2018;64(11):1626-35. https://doi.org/10.1373 /clinchem.2018.289629.

Porteus MH. A new class of medicines through DNA editing. N Engl J Med. 2019 Mar 7;380(10):947-59. https://doi. org/10.1056/NEJMra1800729.

Sakuma T, Yamamoto T. Magic wands of CRISPR-lots of choices for gene knock-in. Cell Biol Toxicol. 2017;33(6):501-5. https://doi.org/10.1007/s10565-017-9409-6.

Sharpe ME. T-cell immunotherapies and the role of nonclinical assessment: the balance between efficacy and pathology. Toxicol Pathol. 2018;46(2):131-46. https://doi.org/10.1177 /0192623317752101.

Song D, Yang D, Powell CA, Wang X. Cell-cell communication: old mystery and new opportunity. Cell Biol Toxicol.
2019;35(2):89-93. https://doi.org/10.1007/s10565-01909470-y.

Tebas P, Stein D, Tang WW, Frank I, Wang SQ, Lee G, et al. Gene editing of CCR5 in autologous CD4 T cells of persons infected with HIV. N Engl J Med. 2014;370(10):901-10. https://doi.org/10.1056/NEJMoa1300662.

Trendowski MR, El Charif O, Dinh PC Jr, Travis LB, Dolan ME. Genetic and modifiable risk factors contributing to cisplatininduced toxicities. Clin Cancer Res. 2019;25(4):1147-55. https://doi.org/10.1158/1078-0432.CCR-18-2244.

Veres A, Gosis BS, Ding Q, Collins R, Ragavendran A, Brand H, et al. Low incidence of off-target mutations in individual CRISPR-Cas9 and TALEN targeted human stem cell clones detected by whole-genome sequencing. Cell Stem Cell. 2014;15(1):27-30. https://doi.org/10.1016/j. stem.2014.04.020.

Wang W, Gao D, Wang X. Can single-cell RNA sequencing crack the mystery of cells? Cell Biol Toxicol. 2018;34(1):1-6. https://doi.org/10.1007/s10565-017-9404-y.

Wu D, Cheng Y, Wang X, CSGT group. Definition of clinical gene tests. Cell Biol Toxicol. 2019;35(2):83-7. https://doi. org/10.1007/s10565-019-09464-w.

Zhu Z, Qiu S, Shao K, Hou Y. Progress and challenges of sequencing and analyzing circulating tumor cells. Cell Biol Toxicol. 2018;34(5):405-15. https://doi.org/10.1007 /s10565-017-9418-5.

Zuo E, Sun Y, Wei W, Yuan T, Ying W, Sun H, et al. Cytosine base editor generates substantial off-target single-nucleotide variants in mouse embryos. Science. 2019, 2019. https://doi. org/10.1126/science.aav9973.

Publisher's note Springer Nature remains neutral with regard to jurisdictional claims in published maps and institutional affiliations. 